\title{
Extensions of interpolation between the arithmetic-geometric mean inequality for matrices
}

\author{
Mojtaba Bakherad, Rahmatollah Lashkaripour ${ }^{*}$ and Monire Hajmohamadi
}

\section{"Correspondence:}

lashkari@hamoon.usb.ac.ir Department of Mathematics, Faculty of Mathematics, University of Sistan and Baluchestan, Zahedan, Iran

\section{Springer}

\begin{abstract}
In this paper, we present some extensions of interpolation between the arithmetic-geometric means inequality. Among other inequalities, it is shown that if $A, B, X$ are $n \times n$ matrices, then

$$
\left\|A X B^{*}\right\|^{2} \leq\left\|f_{1}\left(A^{*} A\right) X g_{1}\left(B^{*} B\right)\right\|\left\|f_{2}\left(A^{*} A\right) X g_{2}\left(B^{*} B\right)\right\|,
$$

where $f_{1}, f_{2}, g_{1}, g_{2}$ are non-negative continuous functions such that $f_{1}(t) f_{2}(t)=t$ and $g_{1}(t) g_{2}(t)=t(t \geq 0)$. We also obtain the inequality

$$
\left\|A B^{*}\right\|^{2} \leq\left\|p\left(A^{*} A\right)^{\frac{m}{p}}+(1-p)\left(B^{*} B\right)^{\frac{s}{1-p}}\right\|\|\|(1-p)\left(A^{*} A\right)^{\frac{n}{1-p}}+p\left(B^{*} B\right)^{\frac{t}{p}} \|,
$$

in which $m, n, s, t$ are real numbers such that $m+n=s+t=1,\|\cdot\|$ is an arbitrary unitarily invariant norm and $p \in[0,1]$.
\end{abstract}

MSC: Primary 47A64; secondary 15A60

Keywords: arithmetic-geometric mean; unitarily invariant norm; Hilbert-Schmidt norm; Cauchy-Schwarz inequality

\section{Introduction and preliminaries}

Let $\mathcal{M}_{n}$ be the $C^{*}$-algebra of all $n \times n$ complex matrices and $\langle\cdot, \cdot\rangle$ be the standard scalar product in $\mathcal{C}^{n}$ with the identity $I$. The Gelfand map $f(t) \mapsto f(A)$ is an isometrical *isomorphism between the $C^{*}$-algebra $C(\operatorname{sp}(A))$ of continuous functions on the spectrum $\operatorname{sp}(A)$ of a Hermitian matrix $A$ and the $C^{*}$-algebra generated by $A$ and $I$.

A norm $\|\cdot\|$ on $\mathcal{M}_{n}$ is said to be unitarily invariant norm if $\|U A V\|=\|A\|$, for all unitary matrices $U$ and $V$. For $A \in \mathcal{M}_{n}$, let $s_{1}(A) \geq s_{2}(A) \geq \cdots \geq s_{n}(A)$ denote the singular values of $A$, i.e. the eigenvalues of the positive semidefinite matrix $|A|=\left(A^{*} A\right)^{\frac{1}{2}}$ arranged in a decreasing order with their multiplicities counted. Note that $s_{j}(A)=s_{j}\left(A^{*}\right)=s_{j}(|A|)$ $(1 \leq j \leq n)$ and $\|A\|=s_{1}(A)$. The Ky Fan norm of a matrix $A$ is defined as $\|A\|_{(k)}=\sum_{j=1}^{k} s_{j}(A)$ $(1 \leq k \leq n)$. The Fan dominance theorem asserts that $\|A\|_{(k)} \leq\|B\|_{(k)}$ for $k=1,2, \ldots, n$ if and only if $\|A\| \leq\|B\|$ for every unitarily invariant norm(see [1], p.93). The HilbertSchmidt norm is defined by $\|A\|_{2}=\left(\sum_{j=1}^{n} s_{j}^{2}(A)\right)^{1 / 2}$, which is unitarily invariant. 
The classical Cauchy-Schwarz inequality for $a_{j} \geq 0, b_{j} \geq 0(1 \leq j \leq n)$ states that

$$
\left(\sum_{j=1}^{n} a_{j} b_{j}\right)^{2} \leq\left(\sum_{j=1}^{n} a_{j}^{2}\right)\left(\sum_{j=1}^{n} b_{j}^{2}\right)
$$

with equality if and only if $\left(a_{1}, \ldots, a_{n}\right)$ and $\left(b_{1}, \ldots, b_{n}\right)$ are proportional [2]. Bhatia and Davis gave a matrix Cauchy-Schwarz inequality as follows:

$$
\left\|A X B^{*}\right\|^{2} \leq\left\|A^{*} A X\right\|\|\| X B^{*} B \| \mid
$$

where $A, B, X \in \mathcal{M}_{n}$. For further information as regards the Cauchy-Schwarz inequality, see [3-5] and the references therein. Recently, Kittaneh et al. [6] extended inequality (1) to the form

$$
\left\|A X B^{*}\right\|^{2} \leq\left\|\left(A^{*} A\right)^{p} X\left(B^{*} B\right)^{1-p}\right\|\|\|\left(A^{*} A\right)^{1-p} X\left(B^{*} B\right)^{p} \|,
$$

where $A, B, X \in \mathcal{M}_{n}$ and $p \in[0,1]$. Audenaert [7] proved that, for all $A, B \in \mathcal{M}_{n}$ and all $p \in[0,1]$, we have

$$
\left\|A B^{*}\right\|^{2} \leq\left\||| p A^{*} A+(1-p) B^{*} B\right\|\|\|\left|(1-p) A^{*} A+p B^{*} B \|\right| .
$$

In [8], the authors generalized inequality (3) for all $A, B, X \in \mathcal{M}_{n}$ and all $p \in[0,1]$ to the form

$$
\left\|A X B^{*}\right\|^{2} \leq\left\|p A^{*} A X+(1-p) X B^{*} B\right\|\|\|(1-p) A^{*} A X+p X B^{*} B \| .
$$

Inequality (4) interpolates between the arithmetic-geometric mean inequality. In [6], the authors showed a refinement of inequality (4) for the Hilbert-Schmidt norm as follows:

$$
\begin{aligned}
\left\|A X B^{*}\right\|_{2}^{2} \leq & \left(\left\|p A^{*} A X+(1-p) X B^{*} B\right\|_{2}^{2}-r^{2}\left\|A^{*} A X-X B^{*} B\right\|_{2}^{2}\right) \\
& \times\left(\left\|(1-p) A^{*} A X+p X B^{*} B\right\|_{2}^{2}-r^{2}\left\|A^{*} A X-X B^{*} B\right\|_{2}^{2}\right),
\end{aligned}
$$

in which $A, B, X \in \mathcal{M}_{n}, p \in[0,1]$ and $r=\min \{p, 1-p\}$. The Young inequality for every unitarily invariant norm states that $\left\|A^{p} B^{1-p}\right\| \leq\|p A+(1-p) B\|$, where $A, B$ are positive definite matrices and $p \in[0,1]$ (see [9] and also [10, 11]). Kosaki [12] extended the last inequality for the Hilbert-Schmidt norm as follows:

$$
\left\|A^{p} X B^{1-p}\right\|_{2} \leq\|p A X+(1-p) X B\|_{2}
$$

where $A, B$ are positive definite matrices, $X$ is any matrix and $p \in[0,1]$. In [13], the authors considered as a refined matrix Young inequality for the Hilbert-Schmidt norm

$$
\left\|A^{p} X B^{1-p}\right\|_{2}^{2}+r^{2}\|A X-X B\|_{2}^{2} \leq\|p A X+(1-p) X B\|_{2}^{2},
$$

in which $A, B$ are positive semidefinite matrices, $X \in \mathcal{M}_{n}, p \in[0,1]$ and $r=\min \{p, 1-p\}$. 
Based on the refined Young inequality (7), Zhao and Wu [14] proved that

$$
\left\|A^{p} X B^{1-p}\right\|_{2}^{2}+r_{0}\left\|A^{\frac{1}{2}} X B^{\frac{1}{2}}-A X\right\|_{2}^{2}+(1-p)^{2}\|A X-X B\|_{2}^{2} \leq\|p A X+(1-p) X B\|_{2}^{2},
$$

for $0<p \leq \frac{1}{2}$ and

$$
\left\|A^{p} X B^{1-p}\right\|_{2}^{2}+r_{0}\left\|A^{\frac{1}{2}} X B^{\frac{1}{2}}-X B\right\|_{2}^{2}+p^{2}\|A X-X B\|_{2}^{2} \leq\|p A X+(1-p) X B\|_{2}^{2},
$$

for $\frac{1}{2}<p<1$ such that $r=\min \{p, 1-p\}$ and $r_{0}=\min \{2 r, 1-2 r\}$.

In this paper, we obtain some operator and unitarily invariant norms inequalities. Among other results, we obtain a refinement of inequality (5) and we also extend inequalities (2), (3) and (5) to the function $f(t)=t^{p}(p \in \mathcal{R})$.

\section{Main results}

In this section, using some ideas of $[6,15]$, we extend the Audenaert results for the operator norm.

Theorem 1 Let $A, B, X \in \mathcal{M}_{n}$ and $f_{1}, f_{2}, g_{1}, g_{2}$ be non-negative continuous functions such that $f_{1}(t) f_{2}(t)=t$ and $g_{1}(t) g_{2}(t)=t(t \geq 0)$. Then

$$
\left\|A X B^{*}\right\|^{2} \leq\left\|f_{1}\left(A^{*} A\right) X g_{1}\left(B^{*} B\right)\right\|\left\|f_{2}\left(A^{*} A\right) X g_{2}\left(B^{*} B\right)\right\| .
$$

Proof It follows from

$$
\begin{aligned}
\left\|A X B^{*}\right\|^{2} & =\left\|B X^{*} A^{*} A X B^{*}\right\| \\
& =s_{1}\left(B X^{*} A^{*} A X B^{*}\right) \\
& =\lambda_{\max }\left(B X^{*} A^{*} A X B^{*}\right) \quad\left(\text { since } B X^{*} A^{*} A X B^{*} \text { is positive semidefinite }\right) \\
& =\lambda_{\max }\left(X^{*} A^{*} A X B^{*} B\right) \\
& =\lambda_{\max }\left(X^{*} f_{1}\left(A^{*} A\right) f_{2}\left(A^{*} A\right) X g_{2}\left(B^{*} B\right) g_{1}\left(B^{*} B\right)\right) \\
& =\lambda_{\max }\left(g_{1}\left(B^{*} B\right) X^{*} f_{1}\left(A^{*} A\right) f_{2}\left(A^{*} A\right) X g_{2}\left(B^{*} B\right)\right) \\
& \leq\left\|g_{1}\left(B^{*} B\right) X^{*} f_{1}\left(A^{*} A\right) f_{2}\left(A^{*} A\right) X g_{2}\left(B^{*} B\right)\right\| \\
& \leq\left\|g_{1}\left(B^{*} B\right) X^{*} f_{1}\left(A^{*} A\right)\right\|\left\|f_{2}\left(A^{*} A\right) X g_{2}\left(B^{*} B\right)\right\| \\
& =\left\|f_{1}\left(A^{*} A\right) X g_{1}\left(B^{*} B\right)\right\|\left\|f_{2}\left(A^{*} A\right) X g_{2}\left(B^{*} B\right)\right\|
\end{aligned}
$$

that we get the desired result.

Corollary 2 If $A, B, X \in \mathcal{M}_{n}$ and $m, n, s$, $t$ are real numbers such that $m+n=s+t=1$, then

$$
\left\|A X B^{*}\right\|^{2} \leq\left\|\left(A^{*} A\right)^{m} X\left(B^{*} B\right)^{s}\right\|\left\|\left(A^{*} A\right)^{n} X\left(B^{*} B\right)^{t}\right\| .
$$

In the next results, we show some generalizations of inequality (3) for the operator norm. 
Corollary 3 Let $A, B \in \mathcal{M}_{n}$ and let $f_{1}, f_{2}, g_{1}, g_{2}$ be non-negative continuous functions such that $f_{1}(t) f_{2}(t)=t$ and $g_{1}(t) g_{2}(t)=t(t \geq 0)$. Then

$$
\left\|A B^{*}\right\|^{2} \leq\left\|p f_{1}\left(A^{*} A\right)^{\frac{1}{p}}+(1-p) g_{1}\left(B^{*} B\right)^{\frac{1}{1-p}}\right\|\left\|(1-p) f_{2}\left(A^{*} A\right)^{\frac{1}{1-p}}+p g_{2}\left(B^{*} B\right)^{\frac{1}{p}}\right\|,
$$

where $p \in[0,1]$.

Proof Applying Theorem 1 for $X=I$, we have

$$
\begin{aligned}
\left\|A B^{*}\right\|^{2} & \leq\left\|f_{1}\left(A^{*} A\right) g_{1}\left(B^{*} B\right)\right\|\left\|f_{2}\left(A^{*} A\right) g_{2}\left(B^{*} B\right)\right\| \\
& =\left\|\left(f_{1}\left(A^{*} A\right)^{\frac{1}{p}}\right)^{p}\left(g_{1}\left(B^{*} B\right)^{\frac{1}{1-p}}\right)^{1-p}\right\|\left\|\left(f_{2}\left(A^{*} A\right)^{\frac{1}{1-p}}\right)^{1-p}\left(g_{2}\left(B^{*} B\right)^{\frac{1}{p}}\right)^{p}\right\|
\end{aligned}
$$

(by Theorem 1)

$$
\leq\left\|p f_{1}\left(A^{*} A\right)^{\frac{1}{p}}+(1-p) g_{1}\left(B^{*} B\right)^{\frac{1}{1-p}}\right\|\left\|(1-p) f_{2}\left(A^{*} A\right)^{\frac{1}{1-p}}+p g_{2}\left(B^{*} B\right)^{\frac{1}{p}}\right\|
$$

(by the Young inequality).

Corollary 4 Let $A, B \in \mathcal{M}_{n}$ and let $f, g$ be non-negative continuous functions such that $f(t) g(t)=t^{2}(t \geq 0)$. Then

$$
\begin{aligned}
\left\|A B^{*}\right\|^{2} \leq & \left\|p f\left(A^{*} A\right)+(1-p) g\left(B^{*} B\right)\right\|^{\frac{1}{2}}\left\|(1-p) f\left(A^{*} A\right)+p g\left(B^{*} B\right)\right\|^{\frac{1}{2}} \\
& \times\left\|p g\left(A^{*} A\right)+(1-p) f\left(B^{*} B\right)\right\|^{\frac{1}{2}}\left\|(1-p) g\left(A^{*} A\right)+p f\left(B^{*} B\right)\right\|^{\frac{1}{2}},
\end{aligned}
$$

where $p \in[0,1]$.

Proof Applying Theorem 1 and the Young inequality we get

$$
\left\|A B^{*}\right\|^{4} \leq\left\|f\left(A^{*} A\right)^{\frac{1}{2}} g\left(B^{*} B\right)^{\frac{1}{2}}\right\|^{2}\left\|g\left(A^{*} A\right)^{\frac{1}{2}} f\left(B^{*} B\right)^{\frac{1}{2}}\right\|^{2}
$$

(by Theorem 1 for $\sqrt{f}$ and $\sqrt{g}$ )

$$
\begin{aligned}
\leq & \left\|f\left(A^{*} A\right)^{p} g\left(B^{*} B\right)^{1-p}\right\|\left\|f\left(A^{*} A\right)^{1-p} g\left(B^{*} B\right)^{p}\right\| \\
& \times\left\|g\left(A^{*} A\right)^{p} f\left(B^{*} B\right)^{1-p}\right\|\left\|g\left(A^{*} A\right)^{1-p} f\left(B^{*} B\right)^{p}\right\|
\end{aligned}
$$

(by inequality (10))

$$
\begin{aligned}
\leq & \left\|p f\left(A^{*} A\right)+(1-p) g\left(B^{*} B\right)\right\|\left\|(1-p) f\left(A^{*} A\right)+p g\left(B^{*} B\right)\right\| \\
& \times\left\|p g\left(A^{*} A\right)+(1-p) f\left(B^{*} B\right)\right\|\left\|(1-p) g\left(A^{*} A\right)+p f\left(B^{*} B\right)\right\|
\end{aligned}
$$

(by the Young inequality).

\section{Some interpolations for unitarily invariant norms}

In this section, applying some ideas of [6], we generalize some interpolations for an arbitrary unitarily invariant norm.

Let $Q_{k, n}$ denote the set of all strictly increasing $k$-tuples chosen from $1,2, \ldots, n$, i.e. $I \in$ $Q_{k, n}$ if $I=\left(i_{1}, i_{2}, \ldots, i_{k}\right)$, where $1 \leq i_{1}<i_{2}<\cdots<i_{k} \leq n$. The following lemma gives some properties of the $k$ th antisymmetric tensor powers of matrices in $\mathcal{M}_{n}$; see [1], p.18. 
Lemma 5 Let $A, B \in \mathcal{M}_{n}$. Then

(a) $\left(\bigwedge^{k} A\right)\left(\bigwedge^{k} B\right)=\bigwedge^{k}(A B)$ for $k=1, \ldots, n$.

(b) $\left(\bigwedge^{k} A\right)^{*}=\bigwedge^{k} A^{*}$ for $k=1, \ldots, n$.

(c) $\left(\bigwedge^{k} A\right)^{-1}=\bigwedge^{k} A^{-1}$ for $k=1, \ldots, n$.

(d) If $s_{1}, s_{2}, \ldots, s_{n}$ are the singular values of $A$, then the singular values of $\wedge^{k} A$ are $s_{i_{1}}, s_{i_{2}}, \ldots, s_{i_{k}}$, where $\left(i_{1}, i_{2}, \ldots, i_{k}\right) \in Q_{k, n}$.

Now, we show inequality (10) for an arbitrary unitarily invariant norm.

Theorem 6 Let $A, B, X \in \mathcal{M}_{n}$ and $\|\cdot \mid\|$ be an arbitrary unitarily invariant norm. Then

$$
\left\|A X B^{*}\right\|^{2} \leq\left\|\left(A^{*} A\right)^{m} X\left(B^{*} B\right)^{s}\right\|\|\|\left(A^{*} A\right)^{n} X\left(B^{*} B\right)^{t} \|,
$$

where $m, n, s, t$ are real numbers such that $m+n=s+t=1$. In particular, if $A, B$ are positive definite, then

$$
\left.\left\|A^{\frac{1}{2}} X B^{\frac{1}{2}}\right\|\right|^{2} \leq\left\|A^{p} X B^{1-p}\right\|\|\| A^{1-p} X B^{p} \|
$$

where $p \in[0,1]$.

Proof If we replace $A, B$ and $X$ by $\bigwedge^{k} A, \bigwedge^{k} B$ and $\bigwedge^{k} X$, their $k$ th antisymmetric tensor powers in inequality (9) and apply Lemma 5 , then we have

$$
\left\|\bigwedge^{k} A X B^{*}\right\|^{2} \leq\left\|\bigwedge^{k}\left(A^{*} A\right)^{m} X\left(B^{*} B\right)^{s}\right\|\left\|\bigwedge^{k}\left(A^{*} A\right)^{n} X\left(B^{*} B\right)^{t}\right\|,
$$

which is equivalent to

$$
s_{1}^{2}\left(\bigwedge^{k} A X B^{*}\right) \leq s_{1}\left(\bigwedge^{k}\left(A^{*} A\right)^{m} X\left(B^{*} B\right)^{s}\right) s_{1}\left(\bigwedge^{k}\left(A^{*} A\right)^{n} X\left(B^{*} B\right)^{t}\right)
$$

Applying Lemma 5(d), we have

$$
\begin{aligned}
\prod_{j=1}^{k} s_{j}\left(A X B^{*}\right) & \leq \prod_{j=1}^{k} s_{j}^{\frac{1}{2}}\left(\left(A^{*} A\right)^{m} X\left(B^{*} B\right)^{s}\right) \prod_{j=1}^{k} s_{j}^{\frac{1}{2}}\left(\left(A^{*} A\right)^{n} X\left(B^{*} B\right)^{t}\right) \\
& \leq \prod_{j=1}^{k} s_{j}^{\frac{1}{2}}\left(\left(A^{*} A\right)^{m} X\left(B^{*} B\right)^{s}\right) s_{j}^{\frac{1}{2}}\left(\left(A^{*} A\right)^{n} X\left(B^{*} B\right)^{t}\right),
\end{aligned}
$$

where $k=1, \ldots, n$. Inequality (13) implies that

$$
\begin{aligned}
\sum_{j=1}^{k} s_{j}\left(A X B^{*}\right) & \leq \sum_{j=1}^{k} s_{j}^{\frac{1}{2}}\left(\left(A^{*} A\right)^{m} X\left(B^{*} B\right)^{s}\right) s_{j}^{\frac{1}{2}}\left(\left(A^{*} A\right)^{n} X\left(B^{*} B\right)^{t}\right) \\
& \leq\left(\sum_{j=1}^{k} s_{j}\left(\left(A^{*} A\right)^{m} X\left(B^{*} B\right)^{s}\right)\right)^{\frac{1}{2}}\left(\sum_{j=1}^{k} s_{j}\left(\left(A^{*} A\right)^{n} X\left(B^{*} B\right)^{t}\right)\right)^{\frac{1}{2}}
\end{aligned}
$$

(by the Cauchy-Schwarz inequality), 
where $k=1, \ldots, n$. Hence

$$
\left\|A X B^{*}\right\|_{(k)}^{2} \leq\left\|\left(A^{*} A\right)^{m} X\left(B^{*} B\right)^{s}\right\|_{(k)}\left\|\left(A^{*} A\right)^{n} X\left(B^{*} B\right)^{t}\right\|_{(k)} .
$$

Now, using the Fan dominance theorem [1], p.98, we get the desired result.

Now, using inequality (12), Theorem 6 and the same argument in the proof of Corollaries 3 and 4, we get the following results; these inequalities are generalizations of the Audenaert inequality (3).

Corollary 7 Let $A, B \in \mathcal{M}_{n}, m, n, s, t$ be real numbers such that $m+n=s+t=1$ and let $\|\cdot \mid\|$ be an arbitrary unitarily invariant norm. Then

$$
\left\|A B^{*}\right\|^{2} \leq\left\|\mid p\left(A^{*} A\right)^{\frac{m}{p}}+(1-p)\left(B^{*} B\right)^{\frac{s}{1-p}}\right\|\|\|(1-p)\left(A^{*} A\right)^{\frac{n}{1-p}}+p\left(B^{*} B\right)^{\frac{t}{p}} \|,
$$

where $p \in[0,1]$.

Corollary 8 Let $A, B \in \mathcal{M}_{n}, m, n, s, t$ be real numbers such that $m+n=s+t=2$ and let $\||\|| \mid$ be an arbitrary unitarily invariant norm. Then

$$
\begin{aligned}
\left\|A B^{*}\right\|^{2} \leq & \left\|p\left(A^{*} A\right)^{m}+(1-p)\left(B^{*} B\right)^{s}\right\|^{\frac{1}{2}}\left\|(1-p)\left(A^{*} A\right)^{m}+p\left(B^{*} B\right)^{s}\right\|^{\frac{1}{2}} \\
& \times\left\|p\left(A^{*} A\right)^{n}+(1-p)\left(B^{*} B\right)^{t}\right\|^{\frac{1}{2}}\left\|(1-p)\left(A^{*} A\right)^{n}+p\left(B^{*} B\right)^{t}\right\|^{\frac{1}{2}}
\end{aligned}
$$

in which $p \in[0,1]$.

Remark 9 If we put $n=m=s=t=1$ in inequality (14), then we obtain the Audenaert inequality (3). Also, if we use inequality (6), Corollaries 7 and 8 , then similar to Corollaries 3 and 4 we get the following inequalities:

$$
\begin{aligned}
\left\|A X B^{*}\right\|_{2}^{2} \leq & \left\|p\left(A^{*} A\right)^{\frac{m}{p}} X+(1-p) X\left(B^{*} B\right)^{\frac{s}{1-p}}\right\|_{2} \\
& \times\left\|(1-p)\left(A^{*} A\right)^{\frac{n}{1-p}} X+p X\left(B^{*} B\right)^{\frac{t}{p}}\right\|_{2},
\end{aligned}
$$

where $A, B \in \mathcal{M}_{n}, m, n, s, t$ are real numbers such that $m+n=s+t=1, p \in[0,1]$ and

$$
\begin{aligned}
\left\|A X B^{*}\right\|_{2}^{2} \leq & \left\|p\left(A^{*} A\right)^{m} X+(1-p) X\left(B^{*} B\right)^{s}\right\|_{2}^{\frac{1}{2}}\left\|(1-p)\left(A^{*} A\right)^{m} X+p X\left(B^{*} B\right)^{s}\right\|_{2}^{\frac{1}{2}} \\
& \times\left\|p\left(A^{*} A\right)^{n} X+(1-p) X\left(B^{*} B\right)^{t}\right\|_{2}^{\frac{1}{2}}\left\|(1-p)\left(A^{*} A\right)^{n} X+p X\left(B^{*} B\right)^{t}\right\|_{2}^{\frac{1}{2}}
\end{aligned}
$$

for $A, B \in \mathcal{M}_{n}$, real numbers $m, n, s, t$, in which $m+n=s+t=2$ and $p \in[0,1]$. These inequalities are generalizations of (4) for the Hilbert-Schmidt norms.

In the following theorem, we show a refinement of inequality (15) for the HilbertSchmidt norm. 
Theorem 10 Let $A, B, X \in \mathcal{M}_{n}$. Then

$$
\begin{aligned}
\left\|A X B^{*}\right\|_{2}^{2} \leq & \left(\left\|p\left(A^{*} A\right)^{\frac{m}{p}} X+(1-p) X\left(B^{*} B\right)^{\frac{s}{1-p}}\right\|_{2}^{2}-r^{2}\left\|\left(A^{*} A\right)^{\frac{m}{p}} X-X\left(B^{*} B\right)^{\frac{s}{1-p}}\right\|_{2}^{2}\right) \\
& \times\left(\left\|(1-p)\left(A^{*} A\right)^{\frac{n}{1-p}} X+p X\left(B^{*} B\right)^{\frac{t}{p}}\right\|_{2}^{2}-r^{2}\left\|\left(A^{*} A\right)^{\frac{n}{1-p}} X-X\left(B^{*} B\right)^{\frac{t}{p}}\right\|_{2}^{2}\right),
\end{aligned}
$$

in which $m, n, s$, tare real numbers such that $m+n=s+t=1, p \in[0,1]$ and $r=\min \{p, 1-p\}$.

Proof Applying inequality (11), we deduce that

$$
\begin{aligned}
\left\|A X B^{*}\right\|_{2}^{2} \leq & \left\|\left(A^{*} A\right)^{m} X\left(B^{*} B\right)^{s}\right\|_{2}\left\|\left(A^{*} A\right)^{n} X\left(B^{*} B\right)^{t}\right\|_{2} \\
= & \left\|\left(\left(A^{*} A\right)^{\frac{m}{p}}\right)^{p} X\left(\left(B^{*} B\right)^{\frac{s}{1-p}}\right)^{1-p}\right\|_{2}\left\|\left(\left(A^{*} A\right)^{\frac{n}{1-p}}\right)^{1-p} X\left(\left(B^{*} B\right)^{\frac{t}{p}}\right)^{p}\right\|_{2} \\
\leq & \left(\left\|p\left(A^{*} A\right)^{\frac{m}{p}} X+(1-p) X\left(B^{*} B\right)^{\frac{s}{1-p}}\right\|_{2}^{2}-r^{2}\left\|\left(A^{*} A\right)^{\frac{m}{p}} X-X\left(B^{*} B\right)^{\frac{s}{1-p}}\right\|_{2}^{2}\right) \\
& \times\left(\left\|(1-p)\left(A^{*} A\right)^{\frac{n}{1-p}} X+p X\left(B^{*} B\right)^{\frac{t}{p}}\right\|_{2}^{2}-r^{2}\left\|\left(A^{*} A\right)^{\frac{n}{1-p}} X-X\left(B^{*} B\right)^{\frac{t}{p}}\right\|_{2}^{2}\right),
\end{aligned}
$$

where $p \in[0,1]$ and $r=\min \{p, 1-p\}$, and the proof is complete.

Theorem 10 includes a special case as follows.

Corollary 11 ([6], Theorem 2.5) Let $A, B, X \in \mathcal{M}_{n}$. Then

$$
\begin{aligned}
\left\|A X B^{*}\right\|_{2}^{2} \leq & \left(\left\|p A^{*} A X+(1-p) X B^{*} B\right\|_{2}^{2}-r^{2}\left\|A^{*} A X-X B^{*} B\right\|_{2}^{2}\right) \\
& \times\left(\left\|(1-p)\left(A^{*} A\right) X+p X B^{*} B\right\|_{2}^{2}-r^{2}\left\|A^{*} A X-X B^{*} B\right\|_{2}^{2}\right),
\end{aligned}
$$

where $p \in[0,1]$ and $r=\min \{p, 1-p\}$.

Proof For $p \in[0,1]$, if we put $m=t=p$ and $n=s=1-p$ in Theorem 10, then we get the desired result.

The next result is a refinement of inequality (5).

Theorem 12 Let $A, B, X \in \mathcal{M}_{n}(\mathcal{C})$ and let $p \in(0,1)$. Then

(i) For $0<p \leq \frac{1}{2}$,

$$
\begin{aligned}
\left\|A X B^{*}\right\|_{2}^{2} \leq & \left(\left\|p A^{*} A X+(1-p) X B^{*} B\right\|_{2}^{2}-r_{0}\left\|\left(A^{*} A\right)^{\frac{1}{2}} X\left(B^{*} B\right)^{\frac{1}{2}}-A^{*} A X\right\|_{2}^{2}\right. \\
& \left.-(1-p)^{2}\left\|A^{*} A X-X B^{*} B\right\|_{2}^{2}\right)^{\frac{1}{2}} \\
& \times\left(\left\|(1-p) A^{*} A X+p X B^{*} B\right\|_{2}^{2}-r_{0}\left\|\left(A^{*} A\right)^{\frac{1}{2}} X\left(B^{*} B\right)^{\frac{1}{2}}-A^{*} A X\right\|_{2}^{2}\right. \\
& \left.-p^{2}\left\|A^{*} A X-X B^{*} B\right\|_{2}^{2}\right)^{\frac{1}{2}} .
\end{aligned}
$$

(ii) For $\frac{1}{2}<p<1$,

$$
\begin{aligned}
\left\|A X B^{*}\right\|_{2}^{2} \leq & \left(\left\|p A^{*} A X+(1-p) X B^{*} B\right\|_{2}^{2}-r_{0}\left\|\left(A^{*} A\right)^{\frac{1}{2}} X\left(B^{*} B\right)^{\frac{1}{2}}-X\left(B^{*} B\right)\right\|_{2}^{2}\right. \\
& \left.-(1-p)^{2}\left\|A^{*} A X-X B^{*} B\right\|_{2}^{2}\right)^{\frac{1}{2}}
\end{aligned}
$$




$$
\begin{aligned}
& \times\left(\left\|(1-p) A^{*} A X+p X B^{*} B\right\|_{2}^{2}-r_{0}\left\|\left(A^{*} A\right)^{\frac{1}{2}} X\left(B^{*} B\right)^{\frac{1}{2}}-X\left(B^{*} B\right)\right\|_{2}^{2}\right. \\
& \left.-p^{2}\left\|A^{*} A X-X B^{*} B\right\|_{2}^{2}\right)^{\frac{1}{2}}
\end{aligned}
$$

where $r=\min \{p, 1-p\}$ and $r_{0}=\min \{2 r, 1-2 r\}$.

Proof The proof of inequality (17) is similar to that of inequality (16). Thus, we only need to prove the inequality (16).

If $0<p \leq \frac{1}{2}$, replacing $A$ and $B$ by $A^{*} A$ and $B^{*} B$ in inequality (8), respectively, we have

$$
\begin{aligned}
\left\|\left(A^{*} A\right)^{p} X\left(B^{*} B\right)^{1-p}\right\|_{2} \leq & \left(\left\|p A^{*} A X+(1-p) X B^{*} B\right\|_{2}^{2}-r_{0}\left\|\left(A^{*} A\right)^{\frac{1}{2}} X\left(B^{*} B\right)^{\frac{1}{2}}-A^{*} A X\right\|_{2}^{2}\right. \\
& \left.-(1-p)^{2}\left\|A^{*} A X-X B^{*} B\right\|_{2}^{2}\right)^{\frac{1}{2}}
\end{aligned}
$$

Interchanging the roles of $p$ and $1-p$ in the inequality (18), we get

$$
\begin{aligned}
\left\|\left(A^{*} A\right)^{1-p} X\left(B^{*} B\right)^{p}\right\|_{2} \leq & \left(\left\|(1-p) A^{*} A X+p X B^{*} B\right\|_{2}^{2}-r_{0}\left\|\left(A^{*} A\right)^{\frac{1}{2}} X\left(B^{*} B\right)^{\frac{1}{2}}-A^{*} A X\right\|_{2}^{2}\right. \\
& \left.-p^{2}\left\|A^{*} A X-X B^{*} B\right\|_{2}^{2}\right)^{\frac{1}{2}}
\end{aligned}
$$

Applying inequalities (11), (18) and (19), we get the desired result.

Corollary 13 Let $A, B \in \mathcal{M}_{n}(\mathcal{C})$ and $p \in(0,1)$. Then

(i) For $0<p \leq \frac{1}{2}$,

$$
\begin{aligned}
\left\|A B^{*}\right\|_{2}^{2} \leq & \left(\left\|p A^{*} A+(1-p) B^{*} B\right\|_{2}^{2}-r_{0}\left\|\left(A^{*} A\right)^{\frac{1}{2}}\left(B^{*} B\right)^{\frac{1}{2}}-A^{*} A\right\|_{2}^{2}\right. \\
& \left.-(1-p)^{2}\left\|A^{*} A-B^{*} B\right\|_{2}^{2}\right)^{\frac{1}{2}} \\
& \times\left(\left\|(1-p) A^{*} A+p B^{*} B\right\|_{2}^{2}-r_{0}\left\|\left(A^{*} A\right)^{\frac{1}{2}}\left(B^{*} B\right)^{\frac{1}{2}}-A^{*} A\right\|_{2}^{2}\right. \\
& \left.-p^{2}\left\|A^{*} A-B^{*} B\right\|_{2}^{2}\right)^{\frac{1}{2}} .
\end{aligned}
$$

(ii) For $\frac{1}{2}<p<1$,

$$
\begin{aligned}
\left\|A B^{*}\right\|_{2}^{2} \leq & \left(\left\|p A^{*} A+(1-p) B^{*} B\right\|_{2}^{2}-r_{0}\left\|\left(A^{*} A\right)^{\frac{1}{2}}\left(B^{*} B\right)^{\frac{1}{2}}-\left(B^{*} B\right)\right\|_{2}^{2}\right. \\
& \left.-(1-p)^{2}\left\|A^{*} A-B^{*} B\right\|_{2}^{2}\right)^{\frac{1}{2}} \\
& \times\left(\left\|(1-p) A^{*} A+p B^{*} B\right\|_{2}^{2}-r_{0}\left\|\left(A^{*} A\right)^{\frac{1}{2}}\left(B^{*} B\right)^{\frac{1}{2}}-\left(B^{*} B\right)\right\|_{2}^{2}\right. \\
& \left.-p^{2}\left\|A^{*} A-B^{*} B\right\|_{2}^{2}\right)^{\frac{1}{2}}
\end{aligned}
$$

where $r=\min \{p, 1-p\}$ and $r_{0}=\min \{2 r, 1-2 r\}$.

Through the following, we would like to obtain upper bound for $\left\|A X B^{*}\right\|$, for every unitary invariant norm.

The following lemma has been shown in [16], and it is considered as a refined matrix Young inequality for every unitary invariant norm. 
Lemma 14 Let $A, B, X \in \mathcal{M}_{n}$ such that $A, B$ are positive semidefinite. Then, for $0 \leq p \leq 1$, we have

$$
\left\|A^{p} X B^{1-p}\right\| \|^{2}+r_{0}(\|A X\|-\|X B\|)^{2} \leq(p\|A X\|+(1-p)\|X B\|)^{2},
$$

where $r_{0}=\min \{p, 1-p\}$.

Proposition 15 Let $A, B, X \in \mathcal{M}_{n}$. Then

$$
\begin{aligned}
\left\|A X B^{*}\right\|^{2} \leq & \left(\left(p\left\|A^{*} A X\right\|+(1-p)\left\|X B^{*} B\right\|\right)^{2}-r_{0}^{2}\left(\left\|A^{*} A X\right\|-\left\|X B^{*} B\right\| \mid\right)^{2}\right)^{\frac{1}{2}} \\
& \times\left(\left((1-p)\left\|A^{*} A X\right\|+p\left\|X B^{*} B\right\|\right)^{2}-r_{0}^{2}\left(\left\|A^{*} A X\right\|-\left\|X B^{*} B\right\|\right)^{2}\right)^{\frac{1}{2}},
\end{aligned}
$$

where $p \in[0,1]$ and $r_{0}=\min \{p, 1-p\}$.

Proof In inequality (20), if we replace $A$ by $A^{*} A$ and $B$ by $B^{*} B$, then we have

$$
\begin{aligned}
\left\|\left(A^{*} A\right)^{p} X\left(B^{*} B\right)^{1-p}\right\| \leq & \left(\left(p\left\|A^{*} A X\right\|+(1-p)\left\|X B^{*} B\right\|\right)^{2}\right. \\
& \left.-r_{0}^{2}\left(\left\|A^{*} A X\right\|-\left\|X B^{*} B\right\|\right)^{2}\right)^{\frac{1}{2}} .
\end{aligned}
$$

Interchanging $p$ with $1-p$ in inequality (21), we get

$$
\begin{aligned}
\left\|\left(A^{*} A\right)^{1-p} X\left(B^{*} B\right)^{p}\right\| \leq & \left(\left((1-p)\left\|A^{*} A X\right\|+p\left\|X B^{*} B\right\|\right)^{2}\right. \\
& \left.-r_{0}^{2}\left(\left\|A^{*} A X\right\|-\left\|X B^{*} B\right\|\right)^{2}\right)^{\frac{1}{2}} .
\end{aligned}
$$

Now applying inequalities (11), (21) and (22) we get the desired inequality.

\section{Conclusions}

Our application of the methods based on the Audenaert results is presented in this paper to the operator norm and so are some interpolations for an arbitrary unitarily invariant norm. Moreover, we refine some previous inequalities as regards the Cauchy-Schwarz inequality for the operator and Hilbert-Schmidt norms.

Acknowledgements

The first author would like to thank the Tusi Mathematical Research Group (TMRG).

Funding

The authors declare that there is no source of funding for this research.

Competing interests

The authors declare that they have no competing interests.

Authors' contributions

The authors contributed equally to the manuscript and read and approved the final manuscript.

\section{Publisher's Note}

Springer Nature remains neutral with regard to jurisdictional claims in published maps and institutional affiliations. 


\section{References}

1. Bhatia, R: Matrix Analysis. Springer, New York (1997)

2. Mitrinović, DS, Pečarić, JE, Fink, AM: Classical and New Inequalities in Analysis. Kluwer Academic, Dordrecht (1993)

3. Bakherad, M: Some reversed and refined Callebaut inequalities via Kontorovich constant. Bull. Malays. Math. Sci. Soc. (2016). doi:10.1007/s40840-016-0364-9

4. Bakherad, M, Moslehian, MS: Reverses and variations of Heinz inequality. Linear Multilinear Algebra 63(10), 1972-1980 (2015)

5. Bakherad, M, Moslehian, MS: Complementary and refined inequalities of Callebaut inequality for operators. Linear Multilinear Algebra 63(8), 1678-1692 (2015)

6. Al-khlyleh, M, Kittaneh, F: Interpolating inequalities related to a recent result of Audenaert. Linear Multilinear Algebra 65(5), 922-929 (2017)

7. Audenaert, KMR: Interpolating between the arithmetic-geometric mean and Cauchy-Schwarz matrix norm inequalities. Oper. Matrices 9, 475-479 (2015)

8. Zou, L, Jiang, Y: A note on interpolation between the arithmetic-geometric mean and Cauchy-Schwarz matrix norm inequalities. J. Math. Inequal. 10(4), 1119-1122 (2016)

9. Ando, T: Matrix Young inequality. Oper. Theory, Adv. Appl. 75, 33-38 (1995)

10. Bakherad, M, Krnic, M, Moslehian, MS: Reverse Young-type inequalities for matrices and operators. Rocky Mt. J. Math. 46(4), 1089-1105 (2016)

11. Hajmohamadi, M, Lashkaripour, R, Bakherad, M: Some extensions of the Young and Heinz inequalities for matrices. Bull. Iranian Math. Soc. (in press)

12. Kosaki, H: Arithmetic-geometric mean and related inequalities for operators. J. Funct. Anal. 156, $429-451$ (1998)

13. Hirzallah, O, Kittaneh, F: Matrix Young inequalities for the Hilbert-Schmidt norm. Linear Algebra Appl. 308, 77-84 (2000)

14. Zho, J, Wu, J: Operator inequalities involving improved Young and its reverse inequalities. J. Math. Anal. Appl. 421 1779-1789 (2015)

15. Hajmohamadi, M, Lashkaripour, R, Bakherad, M: Some generalizations of numerical radius on off-diagonal part of $2 \times 2$ operator matrices. J. Math. Inequal. (in press)

16. Sababheh, M: Interpolated inequalities for unitarily invariant norms. Linear Algebra Appl. 475, $240-250$ (2005)

\section{Submit your manuscript to a SpringerOpen ${ }^{\circ}$ journal and benefit from:}

- Convenient online submission

- Rigorous peer review

- Open access: articles freely available online

- High visibility within the field

- Retaining the copyright to your article

Submit your next manuscript at $>$ springeropen.com 